\title{
Jamming transition in the traffic-flow model with two-level crossings
}

\author{
Takashi Nagatani \\ College of Engineering, Shizuoka University, Hamamatsu 432, Japan \\ (Received 15 April 1993)
}

\begin{abstract}
We investigate the effect of two-level crossings on the traffic jam in the cellular-automaton (CA) model of traffic flow. The CA model is an extended version of the traffic-flow model proposed by Biham, Middleton, and Levine [Phys. Rev. A 46, R6124 (1992)]. Its model is described in terms of the CA on the disordered square lattice with two components: one is the site of three states representing the onelevel crossing and the other is the site of four states representing the two-level crossing. We find that the dynamical jamming transition does not occur when the fraction $c$ of the two-level crossings becomes larger than the percolation threshold $p_{p, c}\left(c>p_{p, c}\right)$. The dynamical jamming transition occurs at higher density $p$ of cars with increasing fraction $c$ of the two-level crossings below the percolation threshold $\left(c<p_{p, c}\right)$. We also present a simple mean-field theory for the jamming transition in traffic flow with twolevel crossings.
\end{abstract}

PACS number(s): 05.70.Jk, 64.60.Ak, 89.40.+k

\section{INTRODUCTION}

Recently, traffic problems have attracted considerable attention [1]. The computer simulation of traffic flow in an entire city is a formidable task since it involves many degrees of freedom. Biham, Middleton, and Levine [2] have proposed a simple cellular-automaton (CA) model to describe a traffic flow in two dimensions. The trafficflow model is given by a three-state CA on the square lattice. Each site either contains a car moving upwards, a car moving to the right, or is empty. They have found that a dynamical jamming transition occurs at the critical density $p=p_{c}\left(p_{c} \approx 0.3-0.4\right)$ with increasing density of cars. The dynamical jamming transition separates between the low-density moving phase in which all cars are moving and the high-density jamming phase in which all cars are stopped. A real-space renormalization-group approach has been applied to the jamming transition [3]. However, the mean-field theory analyzing the jamming transition has not been known until now.

The CA models have been increasingly used in the simulations of complex physical system [4-6]. The CA models provide only some general qualitative problems of the complex system while in other cases useful quantitative information can be obtained.

In real traffic-flow systems, the traffic jam is frequently relaxed by introducing two-level crossings. The jamming single-level crossing prevents cars from crossing its point. By increasing the number of two-level crossings, the traffic jam is avoided even at a high density of cars. The occurrence of a traffic jam strongly depends on the number of two-level crossings.

In this paper, we study the effect of two-level crossings on the traffic jam in the cellular-automaton model of traffic flow. We extend the CA model proposed by $\mathrm{Bi}$ ham, Middleton, and Levine [2] to take into account the two-level crossings. The two-level crossings are randomly introduced on the sites of the square lattice. Our model is described in terms of the CA on the disordered square lattice with two components: one is the site of the single-level crossing and the other is the site of the twolevel crossing. The single-level crossing is described by the three states that either represent a car moving upwards, a car moving to the right, or show an empty state. The two-level crossing is described by the four states that either represent two cars moving upwards and to the right, a car moving upwards, a car moving to the right, or show an empty state. The traffic jam is due to the excluded-volume effect of cars. Without two-level crossings, the traffic jam always occurs above the jamming transition point. However, it is expected that the traffic jam will not occur above the percolation threshold since the infinite path connected by two-level crossings (an infinite cluster of two-level crossings) appears and cars can be free to move on the infinite cluster. We show that the dynamical jamming transition does not occur when the fraction $c$ of the two-level crossings becomes larger than the directed percolation threshold $p_{p, c}$ [7-10]. Below the percolation threshold $\left(c<p_{p, c}\right)$, the jamming transition occurs at higher density $p$ of cars with increasing fraction $c$ of the two-level crossings. We also present a simple mean-field theory for the jamming transition in the CA model with two-level crossings.

The organization of the paper is as follows. In Sec. II, we propose the extended CA model of the traffic flow to take into account the two-level crossings. We describe the computer-simulation method for the extended CA model. In Sec. III, we present the simulation result. We show the dependence of the velocity of cars on the density $p$ of cars and the fraction $c$ of two-level crossings. We show the effect of the two-level crossings on the jamming transition. In Sec. IV, we present a simple mean-field theory for the jamming transition. Finally, Sec. V contains a brief summary.

\section{MODEL AND SIMULATION}

We describe the CA model of traffic flow with twolevel crossings in two dimensions, showing the traffic-jam 
transition and the percolation transition. The CA model is an extended version of the traffic-flow model proposed by Biham, Middleton, and Levine [2]. The CA model takes into account the effect of two-level crossings. The CA model is defined on the square lattice of $n \times n$ sites with periodic boundary conditions. The traffic-flow model is given by the CA model on the square lattice with sites of two components. The one component is the site of the single-level crossing and the other component is the site of the two-level crossing. The two-level crossings are randomly distributed with probability $c$ on the square lattice and the single-level crossing with probability $1-c$. Each site on the single-level crossings either contains an arrow pointing up, an arrow pointing to the right, or is empty. The single-level crossing is described by the three-state CA. Each site on the two-level crossings either contains two arrows pointing up and to the right, an arrow pointing up, an arrow pointing to the right, or is empty. The two-level crossing is described by the fourstate CA. The arrow pointing up represents the car moving up. The arrow pointing to the right represents the car moving to the right. For illustration, Fig. 1 shows the CA model with two-level crossings. The car is inhibited from overlapping on the single-level crossing. However, on the two-level crossing, the car moving up is allowed to overlap the car moving to the right. The movement of cars is controlled by a traffic light. The arrows pointing up move only in even time steps, and the arrows pointing to the right move in odd time steps. On even time steps, if the up nearest-neighbor site is the singlelevel crossing, each arrow pointing up moves one step up unless the up nearest-neighbor site is occupied by the right arrow or the up arrow. If an up arrow is blocked above by another arrow, it does not move, even if the blocking arrow moves out of the site during the same time step. If the up nearest-neighbor site is the two-level crossing, each up arrow moves one step up, unless the up nearest-neighbor site is occupied by the up arrow. If an up arrow is blocked above by the up arrow, it does not move even if the blocking arrow moves out of the site during the same time step. On odd time steps, if the right

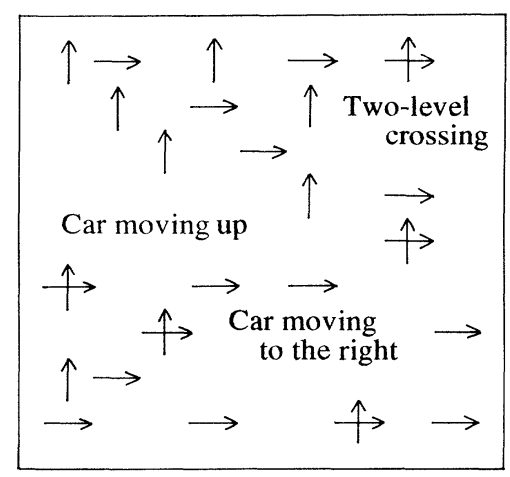

FIG. 1. Schematic illustration of the cellular-automaton model of traffic flow with two-level crossings. The arrow pointing up represents the car moving up. The arrow pointing to the right represents the car moving to the right. The car moving up is allowed to overlap the car moving to the right at the two-level crossing. nearest-neighbor site is the single-level crossing, each arrow pointing to the right moves one step to the right, unless the right nearest-neighbor site is occupied by the right arrow or the up arrow. If a right arrow is blocked by another arrow on the right-hand side, it does not move, even if the blocking arrow moves out of the site during the same time step. If the right nearest-neighbor site is the two-level crossing, each right arrow moves one step to the right unless the right nearest-neighbor site is occupied by the right arrow. If a right arrow is blocked by the right arrow on the right-hand side, it does not move, even if the blocking right arrow moves out of the site during the same time step.

In this model, the traffic problem is reduced to its simplest form. The essential features are maintained. These features include the simultaneous flow of cars in two perpendicular directions in which cars cannot overlap on the single-level crossing, and on the two-level crossing both cars moving up and to the right can overlap. We investigate the effect of the two-level crossings on the traffic jam in this model. In the original model proposed by Biham, Middleton, and Levine [2], the jamming transition is due to the excluded-volume effect of the interaction between the up and right arrows. However, in our model, the jamming transition is relaxed by the reduction of the excluded-volume effect induced by the overlap of cars on the two-level crossings.

We consider the simulation procedure for the CA model explained above. The single-level and two-level crossings are randomly distributed, respectively, with probability $1-c$ and probability $c$. The densities of right and up arrows are given, respectively, by $p_{x}=p / 2$ and $p_{y}=p / 2$ without an overlap of cars, where $p$ is the density of cars. Initially, cars are randomly distributed on the sites of the square lattice without an overlap of cars. The up arrows move or stop in even time steps according to the CA rules explained above. Then, in odd time steps, the right arrows move or stop according to the CA rules. We have performed simulations of the CA model starting with an ensemble of random initial conditions where the system size is $n=15-50, p=0.0-1.0$, and $c=0.0-1.0$. Each run is obtained after 10000 time steps. The total number of arrows of each type is conserved due to the periodic boundary condition. The total number of up arrows in each column and the total number of right arrows in each row are conserved. They give rise to $2 n$ conservation rules.

\section{SIMULATION RESULT}

We present the simulation result obtained by the procedure described in Sec. II. We have obtained the mean velocity of cars for the system size $50 \times 50$ by averaging over 100 runs. Figure 2 shows the plot of the mean velocity $\langle v\rangle$ of cars against the density $p$ of cars for the fraction $c=0.0,0.5,0.6,0.7,0.8$, and 0.9 of two-level crossings. The mean velocity $\left\langle v_{x}\right\rangle(=\langle v\rangle)$ of cars moving to the right equals that $\left\langle v_{y}\right\rangle$ of cars moving up since $p_{x}=p_{y}=p / 2$. The mean velocity $\langle v\rangle$ of cars moving in a unit time interval is defined to be the number of successfully moving arrows divided by the number of ar- 


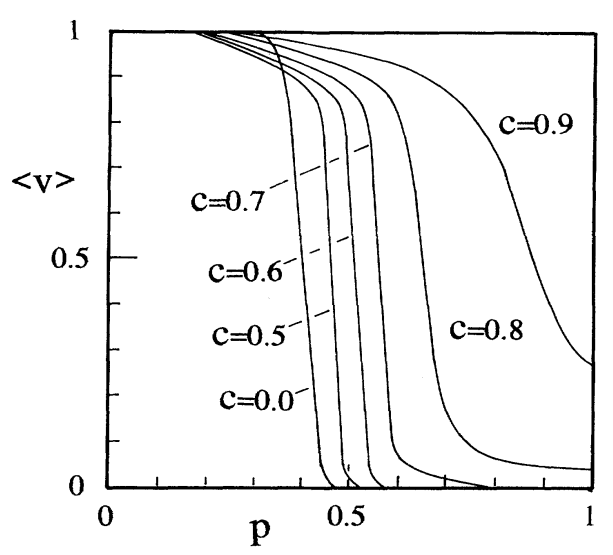

FIG. 2. Plot of the mean velocity $\langle v\rangle$ of cars against the density $p$ of cars for the fraction $c=0.0,0.5,0.6,0.7,0.8$, and 0.9 of two-level crossings.

rows. The velocity $\langle v\rangle$ has a maximum value $\langle v\rangle=1$, indicating that the arrow is never blocked, while $\langle v\rangle$ means that the arrow is stopped and never moves at all. The curve of $c=0.0$ corresponds to the velocity distribution in the original CA model proposed by Biham, Middleton, and Levine [2] with no two-level crossings. With increasing fraction $c$ of two-level crossings, the mean velocity $\langle v\rangle$ becomes zero at higher and higher density $p$. The point in which the velocity becomes zero gives the dynamical jamming transition point. The jamming transition point separates between the moving phase in which cars are moving and the jamming phase in which all cars are stopped. For the cases with the fractions $c=0.8$ and 0.9 , the mean velocity $\langle v\rangle$ of cars never becomes zero, even at $p=1$. Generally, an infinite path connected by the two-level crossings appears when the fraction $c$ of two-level crossings becomes larger than the directed percolation threshold $p_{p, c}=0.7058$ [7-10]. This means that cars can move on the percolating cluster of the two-level crossings. The dynamical jamming transition will depend strongly on the percolation transition. To see the relation between the dynamical jamming transition and the percolation transition, we show the phase diagram between the density $p$ of cars and the fraction $c$ of two-level crossings in Fig. 3. The circles indicate the jamming transition point for the system size $50 \times 50$. The region on the left-hand and upper sides of the transition curve represents the moving phase in which cars move. The region below the transition curve represents the jamming phase in which all cars are stopped. With increasing fraction $c$ of two-level crossings, the jamming transition shifts to the higher density $p$. The traffic jam is relaxed with the increase of the number of two-level crossings. Near the directed percolation threshold $p_{p, c}$ $=0.7058$, the jamming transition point suddenly disappears. This is due to the appearance of the percolating cluster of two-level crossings. On the percolating cluster of two-level crossings, cars are free to move since a car moving up can overlap a car moving to the right on a two-level crossing. The two-level crossings have an important effect on the dynamical jamming transition.

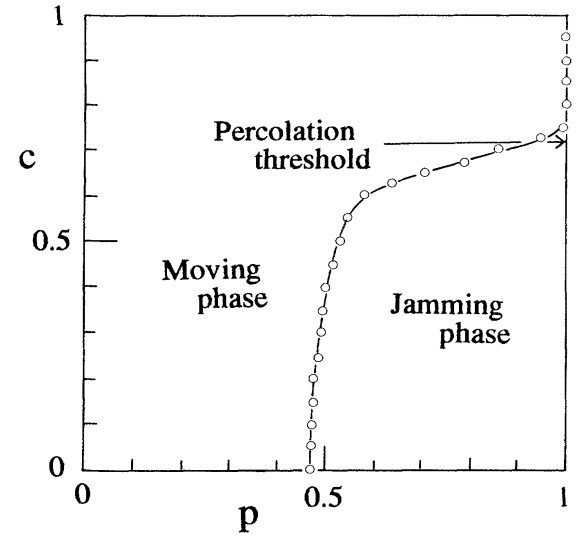

FIG. 3. Phase diagram between the density $p$ of cars and the fraction $c$ of two-level crossings. The region on the left-hand and up sides of the transition curve represents the moving phase in which cars move. The region below the transition curve represents the jamming phase in which all cars are stopped.

Above the percolation threshold, the jamming transition does not occur. Below the percolation threshold, the jamming transition occurs at higher density $p$ of cars with increasing number of two-level crossings.

The jamming transition depends on the system size. In Fig. 4, we plot the transition point $p_{c}$ against the inverse $1 / n$ of the system size for $c=0.0,0.5$, and 0.6. By extrapolation, we estimate the value of the transition point in the limit of an infinite system size. The estimated values $p_{c}$ for $c=0.0,0.5$, and 0.6 are given respectively by $p_{c}=0.42 \pm 0.04,0.49 \pm 0.04$, and $0.53 \pm 0.04$.

The dynamical jamming transition is due to selforganization induced by the excluded-volume effect. The percolation transition is due to the appearance of an infinite cluster of two-level crossings. We show the patterns of cars for the traffic jam in traffic flow with twolevel crossings. Figure 5 shows the typical patterns of configurations of cars for the density $p=0.6$ of cars and the system size $50 \times 50$. Patterns (a) and (b) indicate, respectively, the snapshots obtained for the fraction $c=0.5$

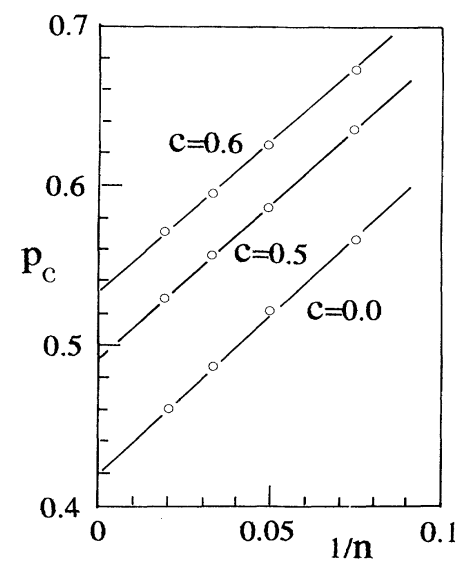

FIG. 4. Plot of the transition point $p_{c}$ against the inverse $1 / n$ of the system size for extrapolation. 


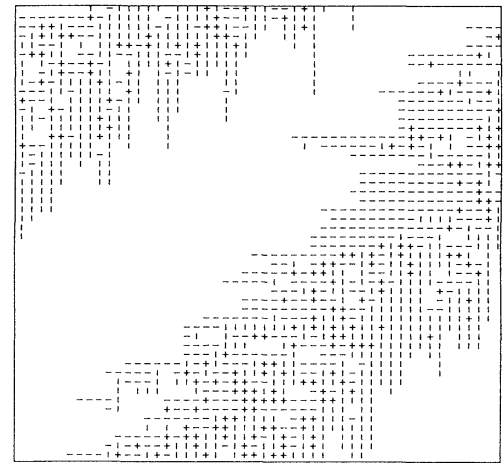

(a)

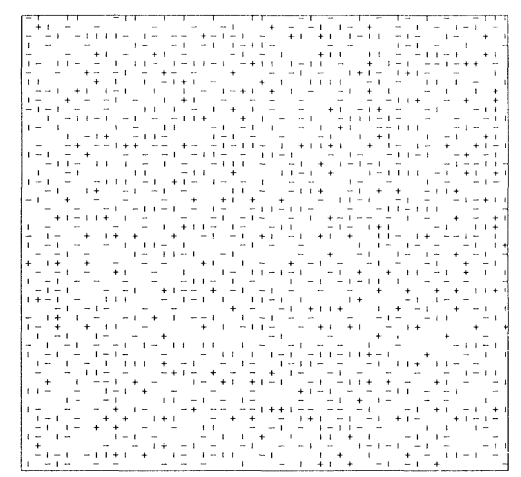

(b)

FIG. 5. Typical patterns of cars for the density $p=0.6$ of cars and the system size $50 \times 50$. The numbers $N_{y}$ and $N_{x}$ of cars moving up and to the right are $N_{y}=754$ and $N_{x}=714$. (a) Pattern of the jamming phase obtained for the fraction $c=0.5$ of two-level crossings. (b) Pattern of the moving phase obtained for the fraction $c=0.8$. The mean velocity is $\langle v\rangle=0.89$.

and 0.8 of two-level crossings. The cars moving up are indicated by the vertical bars. The cars moving to the right are indicated by the horizontal bars. The cross represents the overlap between cars moving to the up and to the right at the two-level crossing. In patterns (a) and (b), the numbers $N_{y}$ and $N_{x}$ of cars moving up and to the

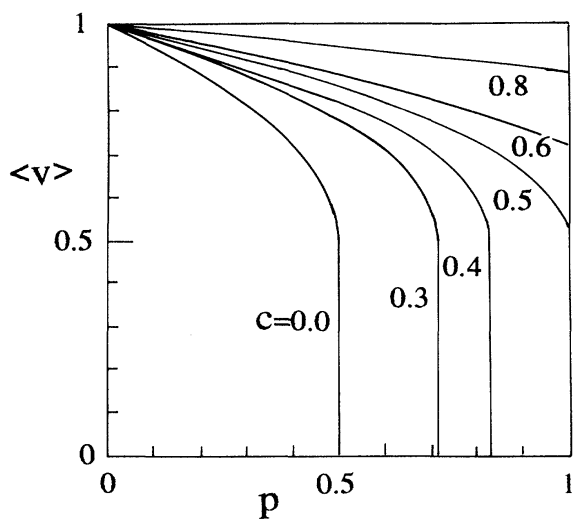

FIG. 6. Plot of the mean velocity $\langle v\rangle$ against the density $p$ of cars obtained from the mean-field theory. right are $N_{y}=754$ and $N_{x}=714$. In pattern (a), the mean velocity is $\langle v\rangle=0.0$. The traffic jam occurs. In pattern (b), where the fraction $c=0.8$ of two-level crossings is larger than $c=0.5$ of pattern (a), the mean velocity is $\langle v\rangle=0.89$. Almost all cars are moving and the traffic jam does not occur. With the increase of two-level crossings, the traffic jam is avoided by allowing the overlap between cars moving up and to the right at the two-level crossing.

\section{MEAN-FIELD THEORY}

We formulate a simple mean-field theory for the jamming transition in traffic flow with two-level crossings. We derive the mean velocities $\left\langle v_{y}\right\rangle$ and $\left\langle v_{x}\right\rangle$ of cars moving up and to the right in a consistent manner. We assume that each right car moves independently with the mean velocity $\left\langle v_{x}\right\rangle$, and each up car moves independently with the mean velocity $\left\langle v_{y}\right\rangle$. A mean time required for a right car to stay on a site is given by $1 /\left\langle v_{x}\right\rangle$. A probability that cars moving to the right prevent a car from moving up is given by $p_{x}(1-c) /\left\langle v_{x}\right\rangle$. Therefore, the mean velocity $\left\langle v_{y}\right\rangle$ of cars moving up is given by the probability that right cars do not prevent an up car from going ahead,

$$
\left\langle v_{y}\right\rangle=1-p_{x}(1-c) /\left\langle v_{x}\right\rangle \text {. }
$$

Similarly, the mean velocity $\left\langle v_{x}\right\rangle$ of cars moving to the right is given by

$$
\left\langle v_{x}\right\rangle=1-p_{y}(1-c) /\left\langle v_{y}\right\rangle \text {. }
$$

Here, we assumed $\left\langle v_{x}\right\rangle \neq 0$ and $\left\langle v_{y}\right\rangle \neq 0$. By setting $p_{x}=p_{y}=p / 2$ and solving Eqs. (1) and (2), we obtain the mean velocity $\langle v\rangle=\left\langle v_{y}\right\rangle=\left\langle v_{x}\right\rangle$ of cars,

$$
\langle v\rangle= \begin{cases}\frac{1+\sqrt{1-2 p(1-c)}}{2}, & \text { if } p<\frac{1}{2(1-c)} \\ 0, & \text { if } p \geq \frac{1}{2(1-c)},\end{cases}
$$

Figure 6 shows the plot of the mean velocity $\langle v\rangle$ against the density $p$ of cars for the fraction $c=0.0,0.3,0.4,0.5$, 0.6 , and 0.8 of the two-level crossings. Above the fraction $c=0.5$, the jamming transition does not occur. The

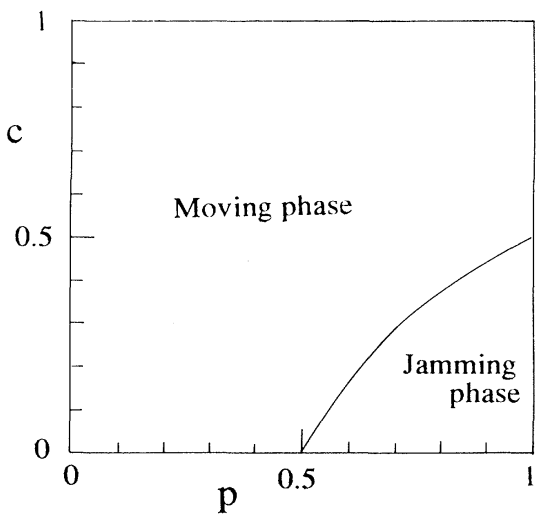

FIG. 7. Phase diagram obtained from the mean-field theory. 
mean velocity $\langle v\rangle$ has a finite value even at $p=1$. Figure 6 is compared with Fig. 2. The solution of $1-2 p(1-c)=0$ gives the jamming transition point,

$$
c=1-\frac{1}{2 p} \text { for } c \leq \frac{1}{2}
$$

For $c>\frac{1}{2}$, the jamming transition does not appear. Figure 7 shows the phase diagram calculated from Eq. (5). This is compared with Fig. 3. The mean-field theory can explain qualitatively the dynamical jamming transition and the percolation transition. However, quantitatively, the result of the mean-field theory is not consistent with the simulation result. This inconsistency is due to neglecting the time-space correlation between cars.

\section{SUMMARY}

We studied the effect of two-level crossings on the traffic jam in the traffic-flow model. We found that the traffic jam does not occur at the fraction $c$ of two-level crossings above the percolation threshold. Also, we showed that the jamming transition occurs at higher density $p$ of cars with increasing fraction $c$ below the percolation threshold. We found the phase diagram representing the moving phase and the jamming phase. We formulated a mean-field theory for the jamming transition.
[1] Transportation and Traffic Theory, edited by N. H. Gatner and N. H. M. Wilson (Elsevier, New York, 1987).

[2] O. Biham, A. A. Middleton, and D. Levine, Phys. Rev. A 46, R6124 (1992).

[3] T. Nagatani (unpublished).

[4] Theory and Applications of Cellular Automata, edited by S. Wolfram (World Scientific, Singapore, 1986).

[5] K. Kaneko, in Formation, Dynamics and Statistics of Patterns, edited by K. Kawasaki, M. Suzuki, and A. Onuki (World Scientific, Singapore, 1990), Vol. 1, p. 1.
[6] P. Bak, C. Tang, and K. Wiesenfeld, Phys. Rev. Lett. 59, 381 (1987).

[7] W. Kinzel, Ann. Israel, Phys. Soc. 5, 425 (1983).

[8] S. Redner, Ann. Israel, Phys. Soc. 5, 447 (1983).

[9] H. E. Stanley, P. J. Reynolds, S. Redner, and F. Family, in Real-Space Renormalization, edited by T. W. Burkhardt and J. M. J. van Leeuwen (Springer, Berlin, 1982), p. 169.

[10] D. Stauffer, Introduction to Percolation Theory (Taylor \& Francis, London, 1985). 